\title{
IMAGE RECOGNITION USING MATLAB SimULINK BLOCKSET
}

\author{
Sagir Lawan and CL Wamdeo \\ Department of Electrical/Electronic Engineering, Nigerian Defence Academy, Kaduna
}

\begin{abstract}
The world over, image recognition are essential players in promoting quality object recognition especially in emergency and search-rescue operation. In this paper precise image recognition system using Matlab Simulink Blockset to detect selected object from crowd is presented. The process involves extracting object features and then recognizes it considering illumination, direction and pose. A Simulink model has been developed to eliminate the tiny elements from the image, then creating segments for precise object recognition. Furthermore, the simulation explores image recognition from the coloured and gray-scale images through image processing techniques in Simulink environment. The tool employed for computation and simulation is the Matlab image processing blockset. The process comprises morphological operation method which is effective for captured images and video. The results of extensive simulations indicate that this method is suitable for application identifying a person from a crow. The model can be used in emergency and search-rescue operation as well as in medicine, information security, access control, law enforcement, surveillance system, microscopy etc.
\end{abstract}

\section{KEYWORDS}

Matlab, Morphological Operations, identification, Target Recognition, Simulink, Video and Image Processing

\section{INTRODUCTION}

Presently, there exists a good number of software for digital image processing in object recognition. Image recognition has varied applications in medicine, information security, access control, law enforcement, surveillance system, microscopy etc. Image processing techniques is used to modify pictures to improve them for recognition. In some cases image processing is used to change images structure by optical, photographic and electronic means. Image processing using digital computers is the most common because digital methods are fast, flexible and precise. Hence, computer algorithms are continuously being developed to achieve better results. The search for more reliable, cheaper and less complex computation led to use of Matlab Simulink Model Blockset for image recognition.

In making operational decisions especially in the military operations in urban terrain, it may be possible to take into consideration image processing to aid in object recognition. This paper aims at identifying important object in images captured from scene using Blockset in Simulink environment of MATLAB. The Video and Image Processing Blockset contains blocks that perform morphological operations such as erosion, dilation, opening and closing. A Simulink model has been developed to eliminate the tiny elements from the image, then creating segments for precise object recognition [1]. The remainder of the paper is organized as follows. Section 2 briefly presents the related research. Section 3 briefly describes on various types of object recognition techniques. Section 4 presents experimental environment. Section 5 covers the results and analysis. Finally, Section 5 draws the conclusion.

DOI: $10.5121 /$ ijcsea.2017.7201 


\section{RELATED RESEARCH}

The idea of target identification has taken various forms prior to image processing. A considerable amount of literature has also been published on digital image processing and intelligent target recognition. In [2], an approach on image/video segmentation was taken in which existing video object segmentation algorithm and morphological operation was used to analyse core operations. Hence, morphology image processing element array and stream processing was used. The author, further stated that control and interconnection between processing element can also be configured. This view is supported by [3], the authors experiment with FPGA using Xlinx system generator to implement MRI image filtering and tour characterization. They focused on processing an image pixel by pixel and in modification of pixel neighbourhoods and the transformation that can be applied to the whole image or partial region. This work was complemented by [4] study of pixel to pixel processing of an image and the modification of pixel transformation to the whole image or only a partial region. However, a big challenge to this approach is feature extraction.

More recent attention has focused on the problem of recognizing of vehicles on a crowed road as contained in [1]. The author found that vehicle counting on roads can reliably be performed using traffic load computation with Matlab Simulink Model Blockset. They stated that Simulink model can be used to detect a particular vehicle in traffic on the road. Their algorithm proves to be an efficient solution for computing traffic load. A broader perspective has been adopted by [5] who argues that segmentation algorithms based on one of two basic properties partition an image based on abrupt change in intensity and regions that are similar according to predefined criteria. Although this is the most comprehensive Simulink image processing, the methods lacks clear details for identification of target. In the same vein, [6] noted the detection and tracking of human as well as vehicles. The paper hitherto have attempted to draw fine distinctions between detection and tracking in three ways (i) automatic target detection and recognition tracking technique, (ii) tracking object across a network and (iii) development of real time communication and message networks.

[7 ] introduced a novel optical encryption and decryption method of gray image based on the computer-generated hologram (CGH). However, in the encryption process, the hologram, which is gotten by Fresnel diffraction from the gray image, is modulated with the chaotic sequence, and the output is encryption image. According to the author in [8] image recognition was achieved through noise reconstruction of holographic images in the form of a system of fringes parallel to the hologram frame boundaries. The author assert that mathematical and physical interpretation is proposed together with an algorithm for reduction of image effect using bicubic splines. Trofimov el at, [9] proposed an improved and a real-time algorithm of computer processing for image recognition using passive $\mathrm{THz}$ image with the aim of concealed object detection. They stated that the algorithm used correlation function between the $\mathrm{THz}$ image and a standard image. The result detect the presence of forbidden objects on the human body..

\section{OBJECT RECOGNITION METHODS}

In the 1970's, object recognition was treated as a 2D pattern recognition problem [2]. The distances between important points where used to recognize known object, however, with technological drive object 2D and 3D objects recognition methods are fully automated. Object recognition methods include holistic matching methods, feature-based (structural) methods and hybrid methods are widely used. 
In both 2D and 3D holistic approach, the complete object region is taken into account as input data into catching system. One of the best examples of holistic methods is Eigenfaces [8] (most widely used method for face recognition). This approach covers face recognition as a twodimensional recognition problem. The process involves insertion of a set of images into a database to create the eigenfaces. Eigenfaces of humans are made by extracting characteristic features from the faces. The input images are normalized to line up the eyes and mouths. When the eigenfaces have been created, each image will be represented as a vector of weights. The weight of the incoming unknown image is found and then compared the weights of those already in the system. If the input image's weight is over a given threshold it is considered to be unidentified. [2].

Another method is described as feature-based (structural) methods: In this methods local features such as eyes, nose and mouth are first of all extracted and their locations and local statistics (geometric and/or appearance) are fed into a structural classifier. A draw back to this method is feature extraction and image restoration [5]. In compliance with need for accurate image recognition, a hybrid method was developed. This method recognized face in both $2 \mathrm{D}$ and $3 \mathrm{D}$ pattern using a combination of both holistic and feature extraction methods.

\section{EXPERIMENTAL ENVIRONMENT}

To date various models for image processing have been developed and introduced. In this paper hybrid method model based design is configured to identify and recognize important object from a crowd using Matlab Simulink Model. The process involves use of Matlab numerical computing environment and matrix manipulations. Simulink model is used to add graphical multi domain simulation and embedded systems. Signal processing is achieved by adapting to the existing algorithms contained in MATLAB programs. Figure 1 shows block diagram of simulation process.

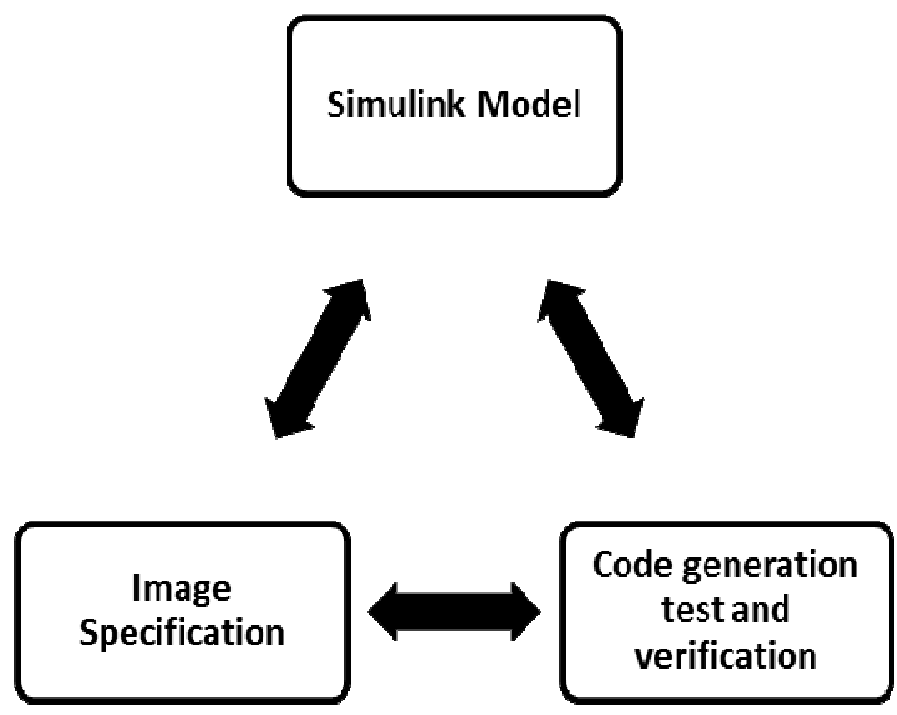

Figure 1: Simulink Model

The basic model design and the fundamental work to read and write images through Simulink is shown in Figures $2-9$. The intensity of the image under experiment is imported From Simulink library browser to create a new Simulink model as indicated in Figure 2. 


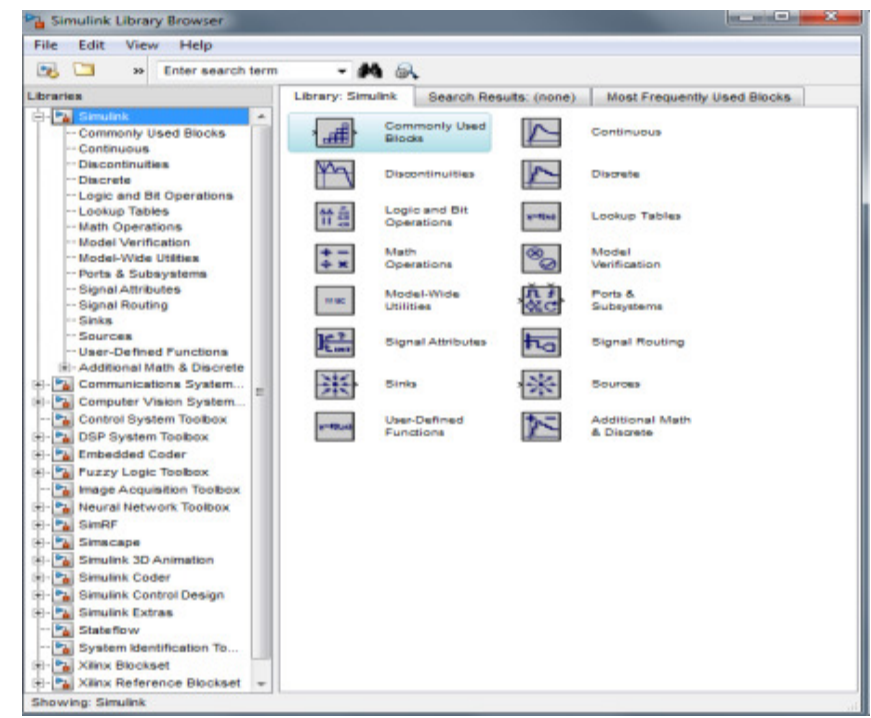

Figure 2: Simulink Library

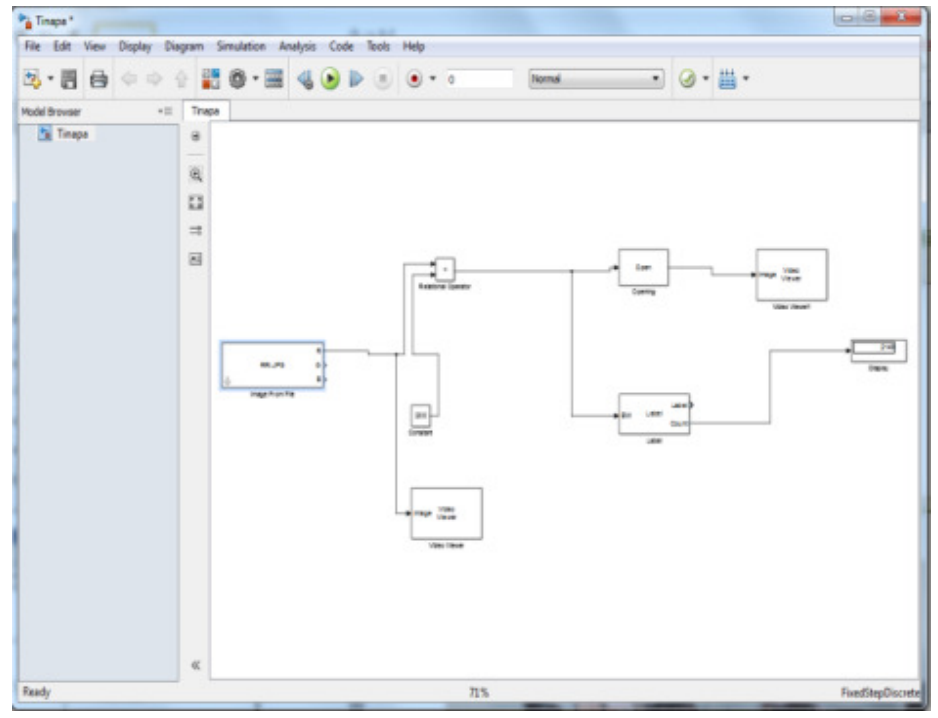

Figure 3: New Model

The values of the block parameters are modified to complain with the specification of the targeted object as demonstrated in Figures 4, 5 and 6. The constant value parameter is set to 200, this defined the threshold. The Rational operator is set to" < " and the configuration parameters is set to solver pane stop time to zero, solver tyre to fixed-step and solver to discrete (no continuous state). The simulated image workspace is illustrated in Figures 7, 10, 13, 16 and19. 
International Journal of Computer Science, Engineering and Applications (IJCSEA) Vol. 7, No. 2, April 2017

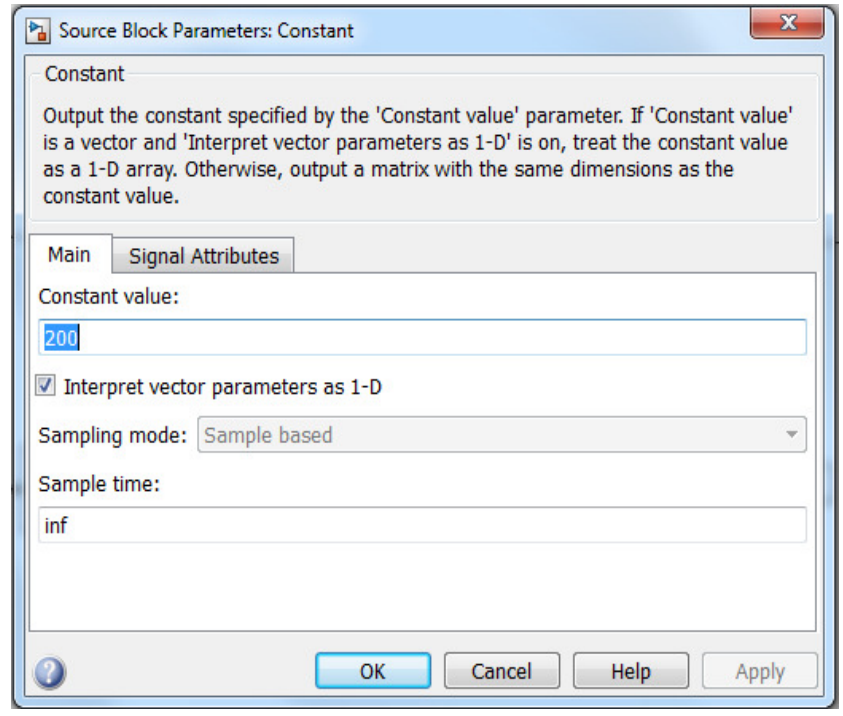

Fig 4: Threshold

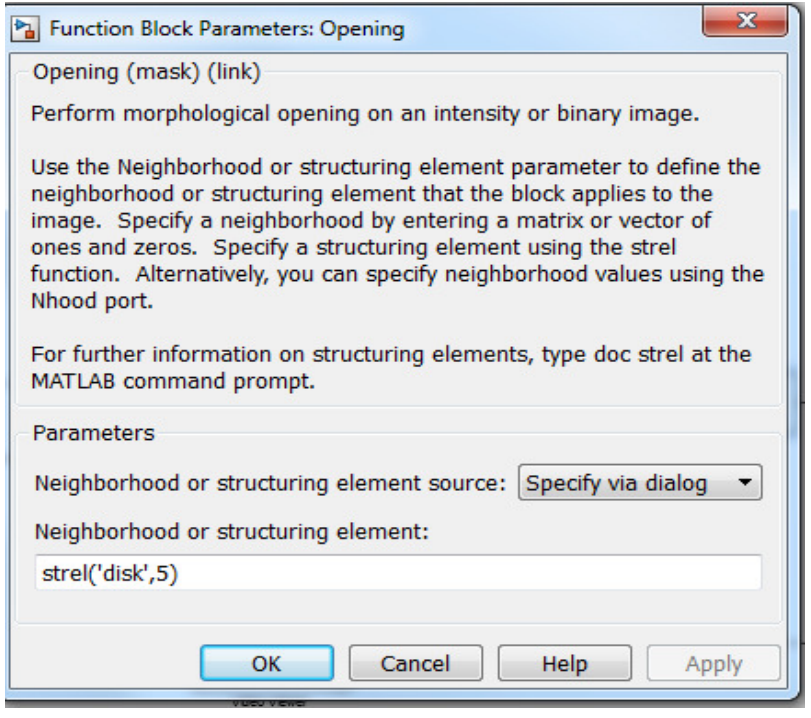

Figure 5: Opening Block 
International Journal of Computer Science, Engineering and Applications (IJCSEA) Vol. 7, No. 2, April 2017

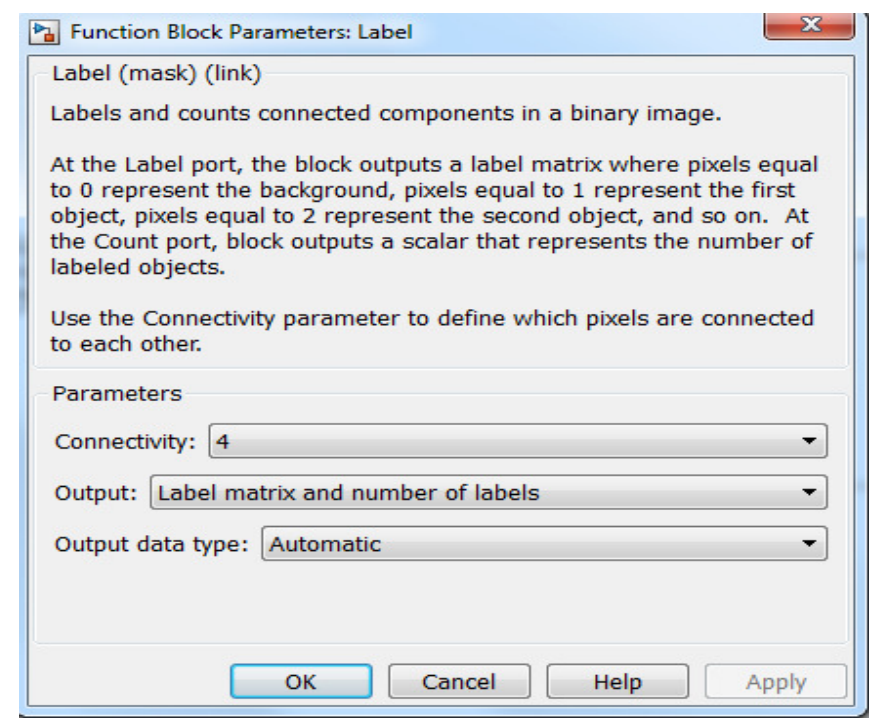

Figure 6: Label Block

\section{RESULTS}

This section describes the results of the simulations conducted; the overall result is presented in terms of: A. coloured and gray-scale image, B. identification and C. boundary performance.

A. Coloured and Gray-Scale Images. The conversion of colour to gray process is done by making all pixels above a certain threshold level white while, others black. The suitable threshold constant taken is 200 . Figures $8,11,14$, and 17 shows results of the image inputs been converted to gray.

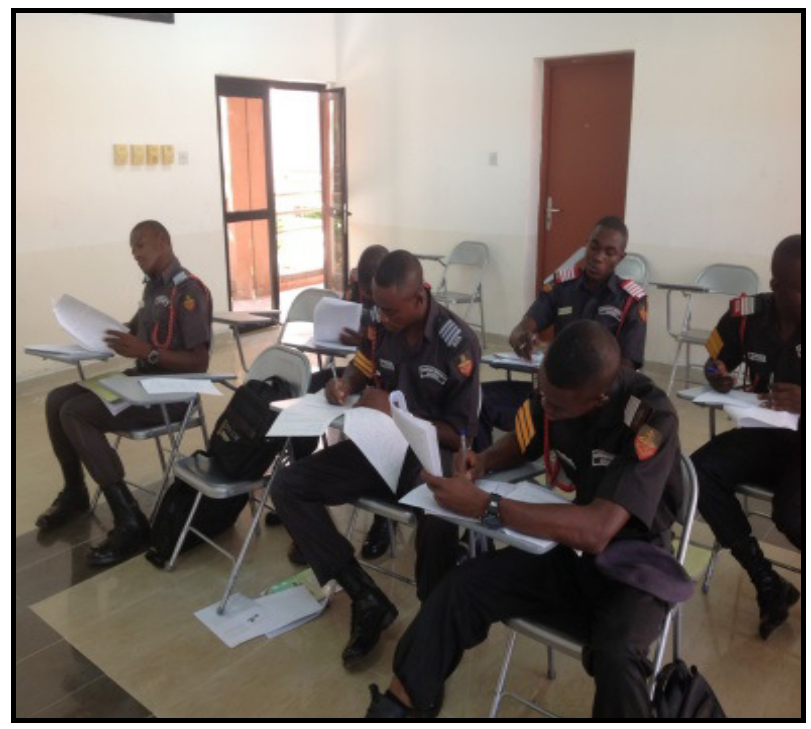

Figure 7: Input Image "Classroom” 


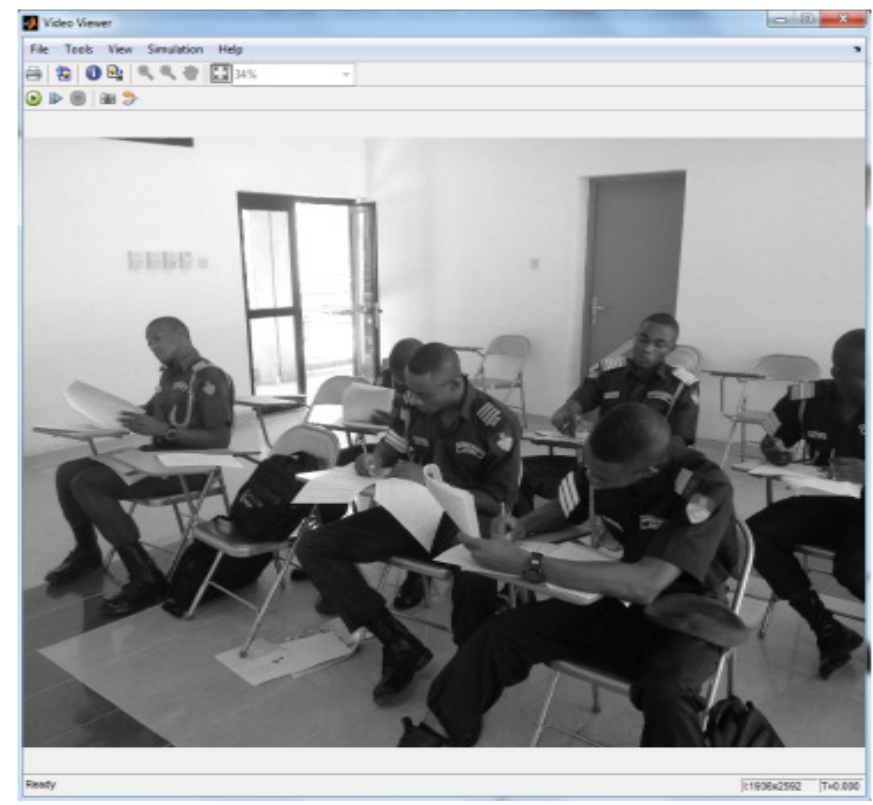

Figure 8: Classroom "Gray"

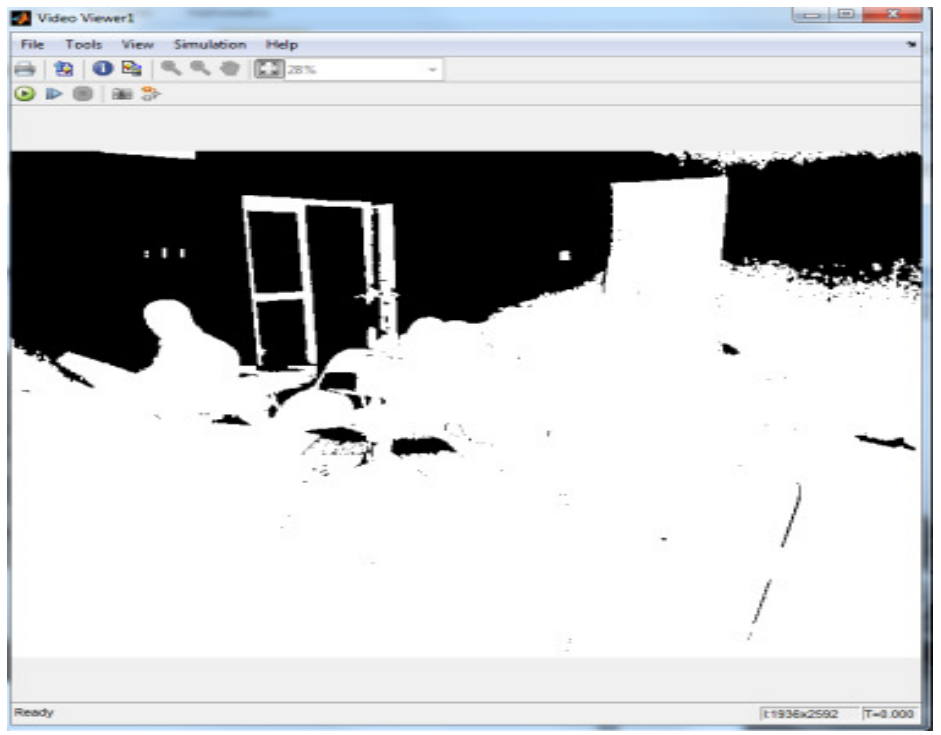

Figure 9: Classroom Object Identified

B. Identification. Identification here is simply masking operation and the main function in the opening block create a circular STREL object with radius of 5 pixels that separate the important objects from the image and from each other at the image. The results of the objects identified in gray are depicted in Figures 9, 12, 15 and 18. 
International Journal of Computer Science, Engineering and Applications (IJCSEA) Vol. 7, No. 2, April 2017

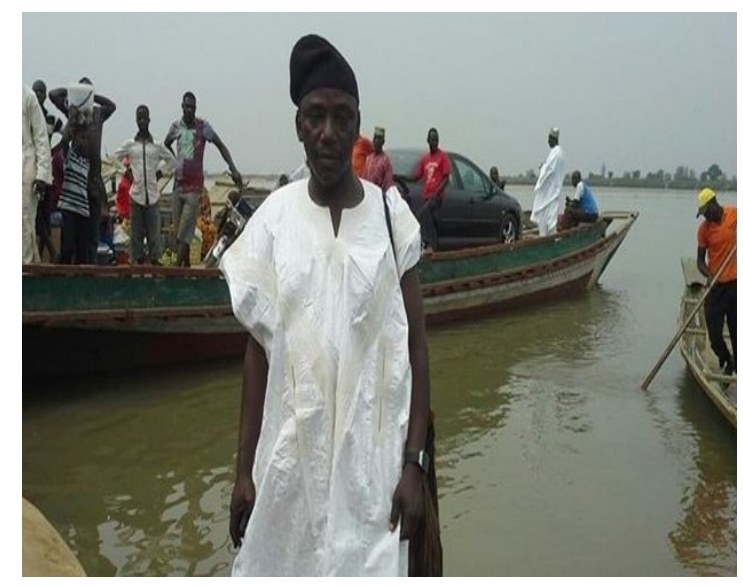

Figure 10: Input image "River side"

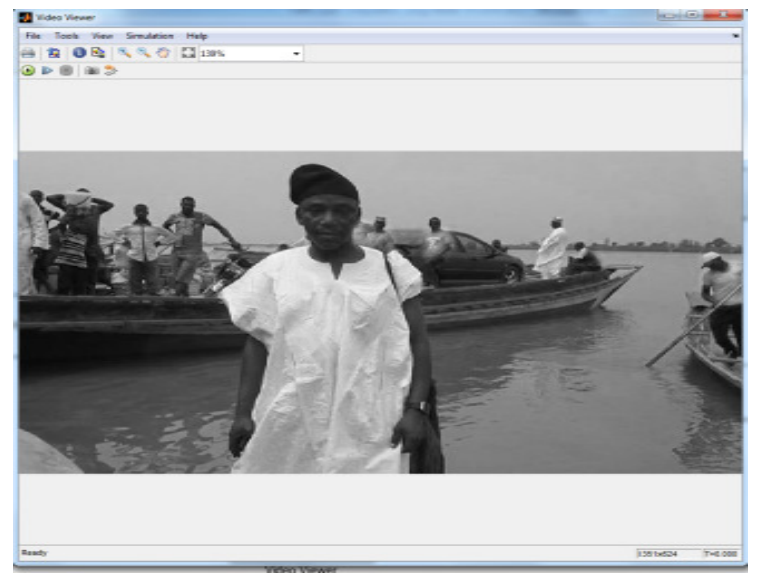

Figure 11: River side Gray

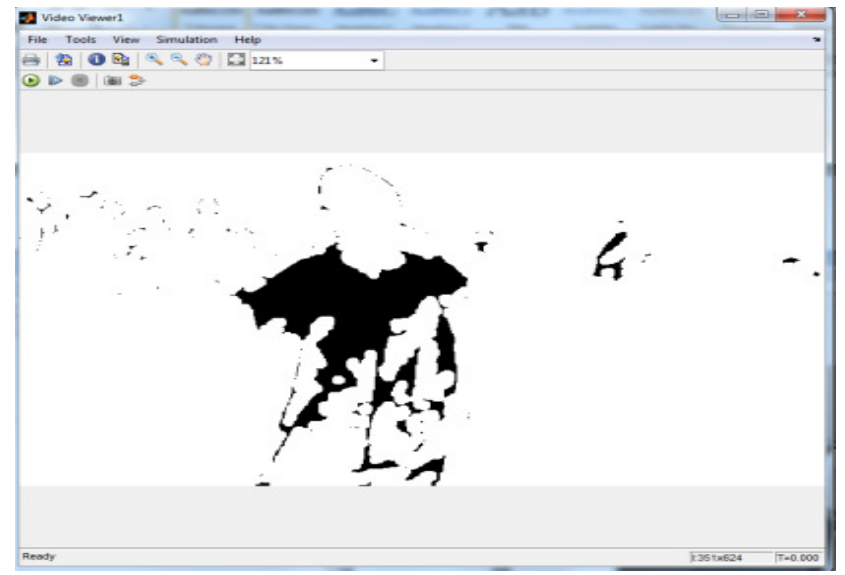

Figure 12: River side Object Identified

C. Boundary. For boundary identification, the image is filtered and masked to ensure better noise suppression and smoothing. The STREL function provided this as illustrated in Figures 9, 12, 15 and 18. 


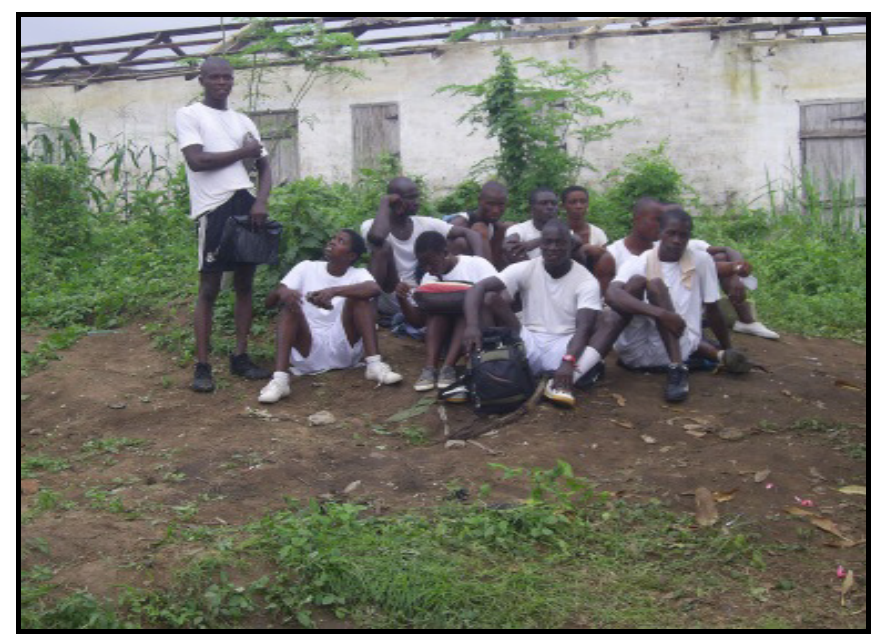

Figure 13: Input image "Youth"

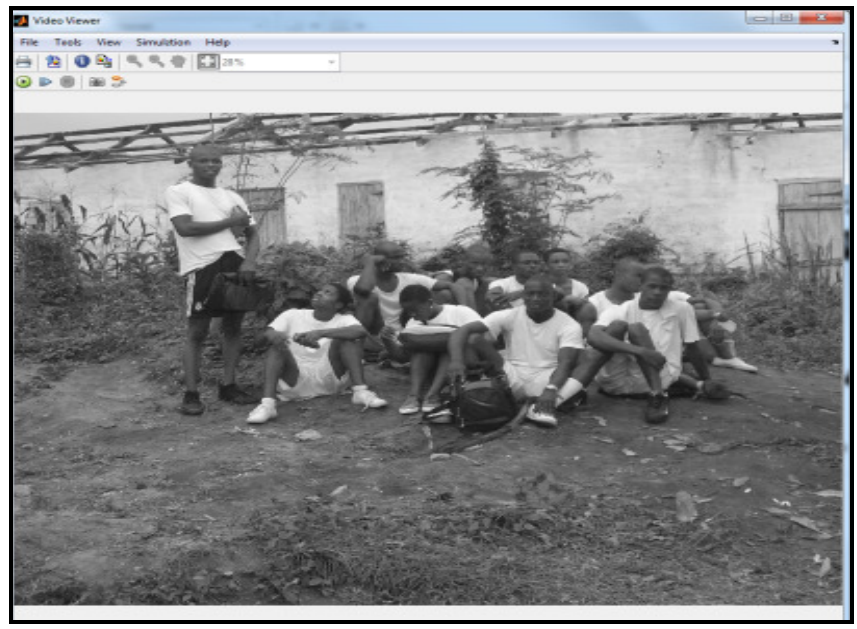

Figure 14: Youth Gray

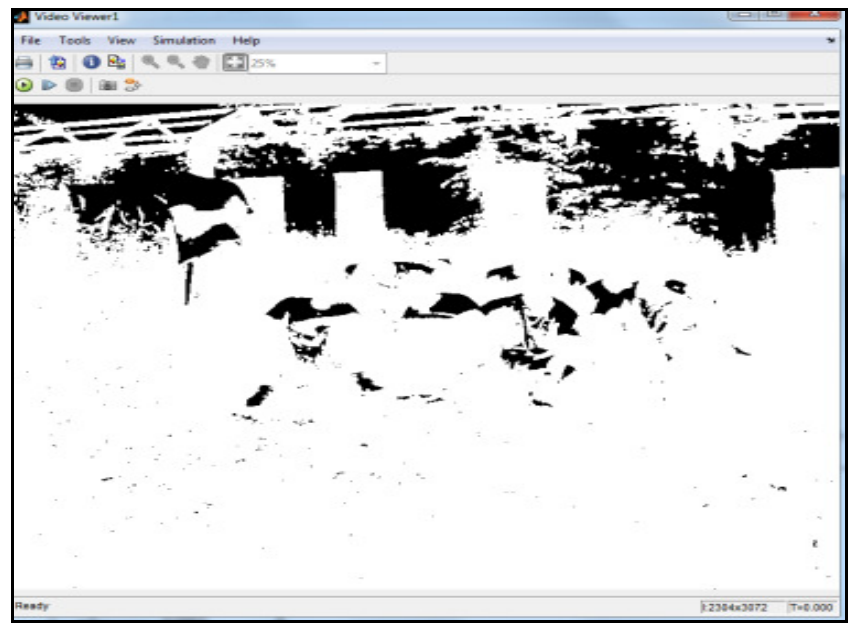


International Journal of Computer Science, Engineering and Applications (IJCSEA) Vol. 7, No. 2, April 2017

Figure 15: Youth Object Identified

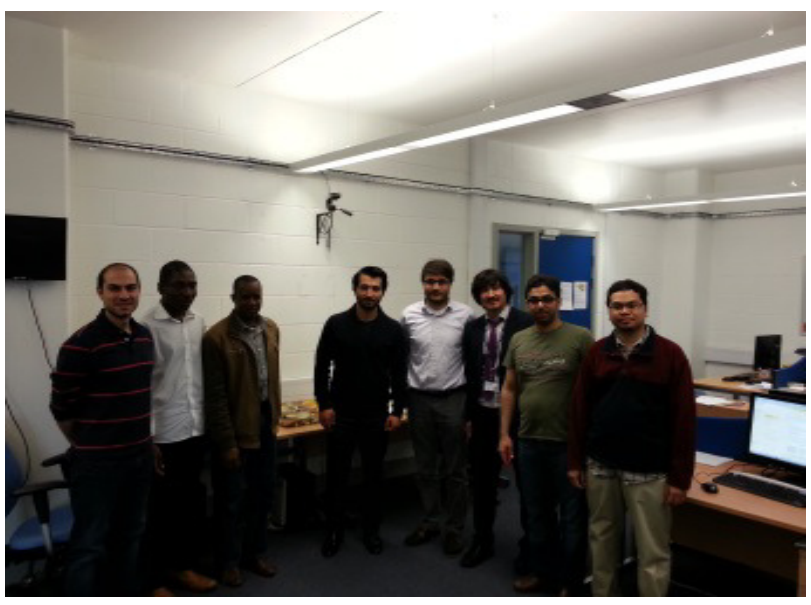

Figure 16: Input image "Lab"

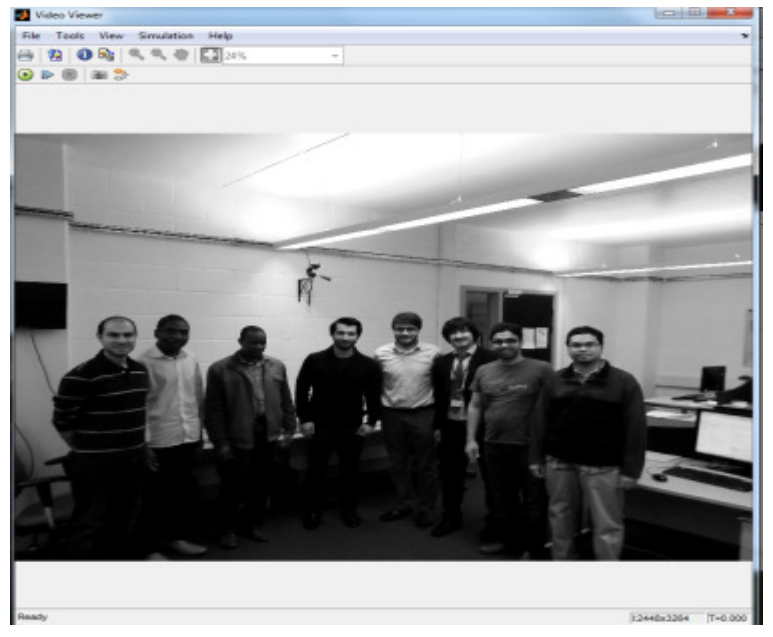

Figure 17: Lab Gray

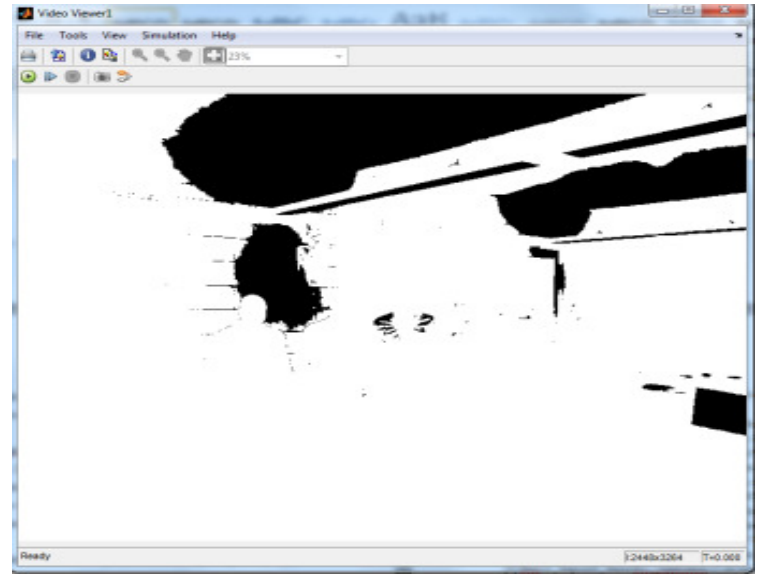

Figure 18: Lab Object Identified 


\section{Conclusion}

In this paper, we presented an image recognition using Matlab Smulink blockset that facilitates flexible identification and recognition of objet in a crowd. Our goal is to separate identified object from many based on configuration and content management of Matlab Smulink blockset. When employing hybrid object identification method for 2D and 3D patterns, the algorithm is easily updated at any time. The proposed method allows reliable and dynamic object identification that will lead to making critical decisions during emergency and search-rescue operations. Feature steps include research on facial feature extraction of human face. Another aspect is integration of the proposed model towards object projection of face images into a linear subspace by reducing dimension.

\section{REFERENCES}

[1] Pratishtha G, Purohit G.N, Adhyana Gupta, "Traffic Load Computer using Matlab Simulink Model Blockset" international Journal of Advanced Research in Computer and Communication Engineering Vol. 2, Issue6, June 2013.

[2] Chien S.Y. ,Chen L.G., "Reconfigurable Morphological Image Processing Accelerator for Video Object Segmentation”, springer, J Sign Process Syst (2011) 62:77-96, DOI 10.1007/s11265-0080311-6

[3] Christe S.A., Vignesh M., Kandaswamy A., "An efficient FPGA implementation of MRI image filtering and tumour characterization using Xilinx system generator”, International Journal of VLSI design \& Communication Systems (VLSICS), Vol.2 ,No.4, December 2011

[4] Suthar A. C., Vayada M., Patel C. B., Kulkarni G.R., Hardware Software co-simulation for Image Processing Applications", IJCSI International Journal of Computer Science Issues, Vol. 9, Issue 2, No 2, March 2012

[5] Al-amri S.S., Kalyankar N.V., Khamitkar S.D, "Image Segmentation by Using Threshold Techniques", journal of computing, volume 2, issue 5, may 2010, ISSN 2151-9617 [6]Shinde B., Mhaske D., Dani A.R., "Study of Image Processing, Enhancement and Restoration", IJCSI International Journal of Computer Science Issues, Vol. 8, Issue 6, No 3, November 2011

[7] Mahalanobis A., Cannon J., Stanfill S.R., Muise R., Martin L., "Network Video Image Processing for Security, Surveillance, and Situational Awareness", Proc. SPIE 5440, Digital Wireless Communications VI,1 (August 10, 2004); doi:10.1117/12.548981

[8] Ren, Hui, Jun Wang, and Qiong-hua Wang. "Optical Encryption of Gray Image Based on the Computer Generated Hologram and Logical Modulation." Proc. of SPIE Vol. Vol. 10250. 2017.

[9] Dyomin, V., and A. Olshukov. "Improvement of the Quality of Reconstructed Holographic Images by Extrapolation of Digital Holograms." Russian Physics Journal 58.10 (2016).

[10] Trofimov, Vyacheslav A., et al. "Concealed object detection using the passive THz image without its viewing." SPIE Defense+ Security. International Society for Optics and Photonics, 2016. 